\title{
Development and Overview of CPAS Sasquatch Airdrop Landing Location Predictor Software
}

\author{
Kristin J. Bledsoe ${ }^{1}$ \\ Jacobs Engineering, Houston, TX, 77058 \\ Michael A. Bernatovich ${ }^{2}$ \\ NASA Johnson Space Center, Houston, TX 77058
}

The Capsule Parachute Assembly System (CPAS) is the parachute system for NASA's Orion spacecraft. CPAS is currently in the Engineering Development Unit (EDU) phase of testing. The test program consists of numerous drop tests, wherein a test article rigged with parachutes is extracted from an aircraft. During such tests, range safety is paramount, as is the recoverability of the parachutes and test article. It is crucial to establish a release point from the aircraft that will ensure that the article and all items released from it during flight will land in a designated safe area. The Sasquatch footprint tool was developed to determine this safe release point and to predict the probable landing locations (footprints) of the payload and all released objects. In 2012, a new version of Sasquatch, called Sasquatch Polygons, was developed that significantly upgraded the capabilities of the footprint tool. Key improvements were an increase in the accuracy of the predictions, and the addition of an interface with the Debris Tool (DT), an in-flight debris avoidance tool for use on the test observation helicopter. Additional enhancements include improved data presentation for communication with test personnel and a streamlined code structure. This paper discusses the development, validation, and performance of Sasquatch Polygons, as well as its differences from the original Sasquatch footprint tool.

\section{Nomenclature}

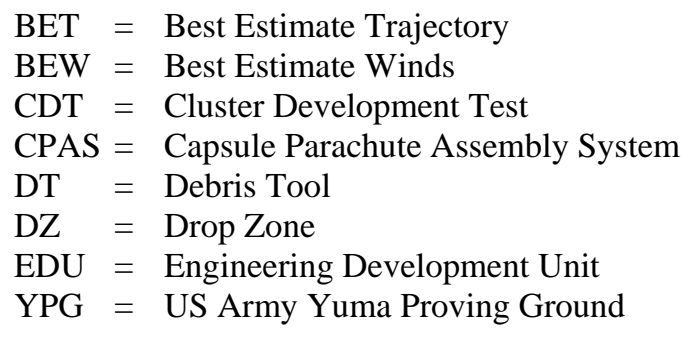

\section{Introduction}

$\mathrm{T}$ HE Capsule Parachute Assembly System (CPAS) is the parachute system for the Orion capsule. CPAS is in the Engineering Development Unit (EDU) phase of testing meant to understand, tune, and finalize the design of a human-rated parachute system. The primary method of testing the CPAS parachutes is by performing drop tests, wherein a test article rigged with parachutes is extracted from an aircraft. Parachutes are deployed in nominal or offnominal sequences to meet the test objectives. Once the test article has landed, the article and all parachutes are recovered for hardware inspection and data analysis. Most CPAS airdrop tests are conducted at the US Army Yuma Proving Ground (YPG).

Range safety must be considered on a test of this type. The test article must land in a safe location on the drop zone, away from observers and buildings. For the protection of the recovery team, CPAS test article, and other CPAS

\footnotetext{
${ }^{1}$ Analysis Engineer, GN\&C, Aerosciences, and Tech Transfer/EPO, 2224 Bay Area Blvd, Houston, TX, AIAA Member.

${ }^{2}$ CPAS Analysis Lead, MC DM4, 2101 NASA Parkway, Houston, TX, AIAA Member.
} 
hardware, an area that has been plowed clear of brush is targeted. In addition, numerous parachutes are deployed during a test and released from the test article creating debris in the form of individual parachutes, clusters of parachutes connected by confluence fitting, mortar lids and sabots, and deployment bags. This debris falls separately from the test article and can be a hazard to assets on the ground and in the air.

In order to ensure the safety of the test team, hardware, and anyone or anything in the area of the drop zone, the regions (i.e. footprints) that bound the landing locations of all of the test article components must be predicted. This is highly dependent on the winds of the day through the altitude range the test article descends. A footprint predictor tool called Sasquatch was developed and used through the first two generations of CPAS testing. Sasquatch perturbed the simulated trajectory of the test article based on the winds of the day to determine the latitude and longitude at which the test article should be extracted from the aircraft at a given altitude in order to ensure a safe footprint. Sasquatch used simplified assumptions, such as a constant rate of descent for each object and horizontal motion aligned with the wind. Dispersion circles designed to account for uncertainties in winds and descent rate were output, centered on each object's calculated landing location. Sasquatch was successfully used on 16 drop tests between December 2007 and February 2012.

As CPAS moved into the EDU phase of testing, the drop tests became more complex. More objects had to be tracked, including mortar lids and sabots and the test article delivery cradle. Chase aircraft, including helicopters and small airplanes, were assigned to take videos and still photographs of the test. These additional hazards and increased complexity required an improved footprint predictor.

Sasquatch Polygons was developed primarily to increase the accuracy of the predictions and to provide an interface with the newly developed Debris Tool (DT), an in-flight debris avoidance tool for use on the test-observation helicopter. Additional enhancements included improved data presentation for communication with test personnel and a streamlined code structure.

\section{History of Sasquatch}

The original version of Sasquatch used a simple, constant rate of descent model to propagate each object's trajectory. The objects moved horizontally at the velocity of the wind, neglecting horizontal drag and inertia. Sasquatch used constant uncertainty values on an object's time of flight, wind magnitude, and the position where the payload was to be extracted from the carrier aircraft in its computation of the dispersion circle's radius. Sample output footprints are shown in Figure 1.

Although this version of the tool provided well-correlated predictions for early tests, the higher altitudes and new debris objects in the EDU phase of testing required higher fidelity predictions. The constant uncertainty values yielded dispersion circles that sometimes severely limited the altitude at which the test article could be released. For a test to be conducted, the footprint was required to have all dispersions stay within the drop zone range limits, with the majority of the payload dispersion circle contained on the cleared area. Even on a day with moderate winds, the dispersions circle areas became unacceptable when releasing the payload from 20,000 ft or higher. It was widely agreed that the circular dispersions and constant uncertainty values were too conservative.

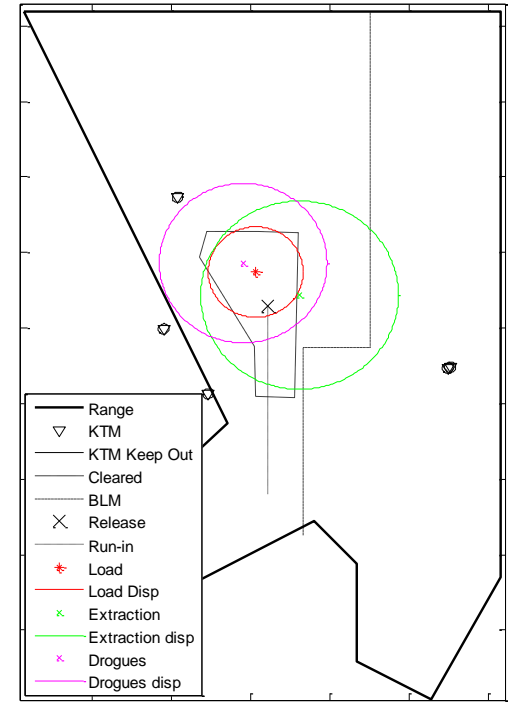

Figure 1. Example Sasquatch dispersion areas. A better understanding of the possible landing locations for all test objects was required. Sasquatch Polygons was developed to address the need for increased precision in the predictions.

\section{Sasquatch Polygons Trajectory Propagation}

The trajectory of an object in a drop test consists of a gravity turn after aircraft extraction followed by a descent with a constant-rate vertical velocity component and horizontal motion due to winds. The original Sasquatch code used a simple Newtonian solver with a constant time step to determine an object's trajectory. Although sufficiently accurate for the CPAS tests at the time, this method was prone to increased error when used for higher altitude drop tests or with a wind profile containing shears. The first step in improving Sasquatch was to replace the simple assumption with a more accurate approach to propagating the trajectories.

Sasquatch Polygons uses MATLAB's ODE45 function, a dynamic time-step, Runge-Kutta differential equation solver. This approach is more consistently accurate than its predecessor since it uses a 3-DOF set of differential equations to propagate the object from its initial state. For mortar components, the velocity is initialized based on the

American Institute of Aeronautics and Astronautics 
known mortar ejection velocity, whereas the original Sasquatch tool used an approximate calculation of the distance that a mortar-deployed object was "thrown" to account for the imparted velocity.

The new approach used to propagate the objects' trajectories is slower than the previous method, since it is more computationally intensive. However, it has been tuned to balance accuracy and run time in a way that consistently integrates well with CPAS drop test operations.

\section{Determination of Footprint Bounds}

The highest priority in developing Sasquatch Polygons was to improve the dispersions on the landing location for each object in the footprint. Both CPAS and test range flight rules require that the dispersion areas cover only safe locations. For the primary test article, that means the dispersion area must remain entirely or mostly on the cleared area of the drop zone. For other objects, the dispersion areas may not cross the highway east of the drop zone, unless a waiver has been obtained. The goal of improving the dispersions was to ensure that footprint-based decisions, such as extraction altitude, are made using the best predictions possible with extra conservatism removed.

A new method of generating dispersion areas was developed for Sasquatch Polygons. Multiple trajectories are propagated for each object, with each trajectory being uniquely perturbed by dispersing factors that most influence the trajectory.

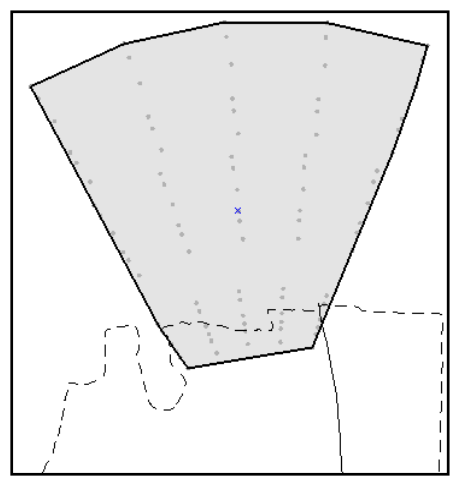

Figure 2. Parametric sweep.

The unique perturbation on each trajectory is defined by a predefined parametric sweep of factors or biases on these key factors. The landing locations of all resulting trajectories are combined to establish the boundary of the dispersion area (Fig. 2). The polygonal shape of these dispersion areas, also called "fans," gives Sasquatch Polygons its name.

Variables currently included in the parametric sweep to generate the dispersion fans are wind speed, wind direction, release altitude, release location, and drag area; other parameters can be added if necessary.

The amount each parameter is varied is based on past test data. A statistical assessment was performed on weather balloon data from previous tests at YPG to determine appropriate dispersions on the wind speed and direction. Winds

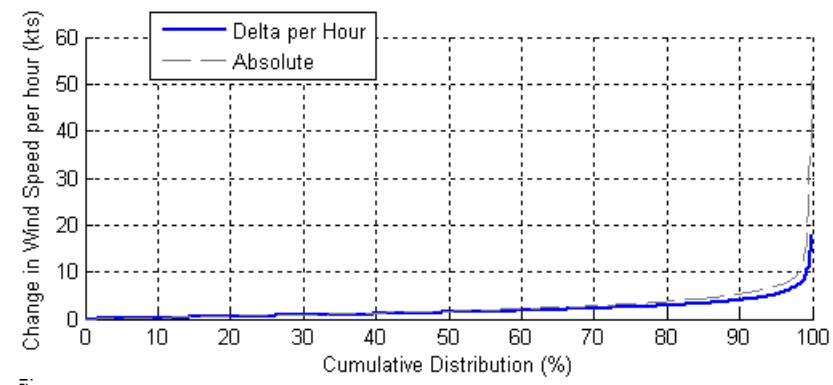

\begin{tabular}{|l|l|l|}
\hline \multicolumn{4}{|l|}{ Change per Hour } \\
\hline Percentile (\%) & Speed (kts) & Dir (deg) \\
\hline 75 & 2.5 & 12.5 \\
\hline 95 & 5.2 & 33.4 \\
\hline 99 & 8.8 & 62.4 \\
\hline 99.7 & 12.75 & 105.5 \\
\hline Mean & 1.87 & 10.2 \\
\hline Absolute Change & \multicolumn{2}{|l}{} \\
\hline Percentile (\%) & Speed (kts) & Dir (deg) \\
\hline 75 & 3.2 & 16.7 \\
\hline 95 & 7 & 42.8 \\
\hline 99 & 14.7 & 100.2 \\
\hline 99.7 & 34 & 144.5 \\
\hline Mean & 2.56 & 13.54 \\
\hline
\end{tabular}

Figure 3. Cumulative distribution of wind deltas.

over the course of a given morning were compared to determine

Table 1. Wind deltas.

the manner and magnitude in which they would trend. Specifically, it is critical to identify a specific change in wind magnitude and direction that accounts for wind persistence. The results for Yuma Proving Ground are shown in Table 1 and Fig. 3.

American Institute of Aeronautics and Astronautics 


\section{Verification and Validation of Results}

\section{A. Comparison with Sasquatch}

During its development Sasquatch Polygons output was compared with that from Sasquatch from past tests, both to verify the results of the new tool and show the improvements in predictions. The expected result was that the nominal landing location predicted for each object would be similar between the two, while the dispersion polygons would be smaller than the circles from the original Sasquatch.

Figure 4 shows a comparison of landing locations and dispersions of selected objects from the CDT-3-4 test. For both runs, the payload (in red) was targeted to land in the optimum location in the cleared area (the irregular shape with a dashed outline). The nominal landing locations of each debris object is offset between the two runs, but the difference is within expectations. The size and shape of the dispersions for each object are notably different. They cover similar areas, but the polygons are typically smaller. The vertex regions of the polygons represent the parametric sweep cases that have

multiple factors being dispersed. For example, the case that defines the extreme left part of the footprint toe is based on a maximum wind speed bias, shifted wind direction, and maximum possible object drag area. These cases are outside of the circle in many cases and are considered the least likely parts of the footprint to expect the object to land. Similar results were seen on other comparisons.

\section{B. Mock Day of Test Predictions}

To determine how Sasquatch Polygons would perform on a test day, runs were conducted using the final set of weather data received on a given test morning and the release point specified by Sasquatch on that test day. The resulting footprints were compared to the actual landing locations. If the actual landing location fell within the corresponding footprint, the prediction was deemed successful.

For example, the study of CDT-3-4 showed that all of the objects landed within their respective footprints. Most objects

landed close to the nominal, or undispersed,

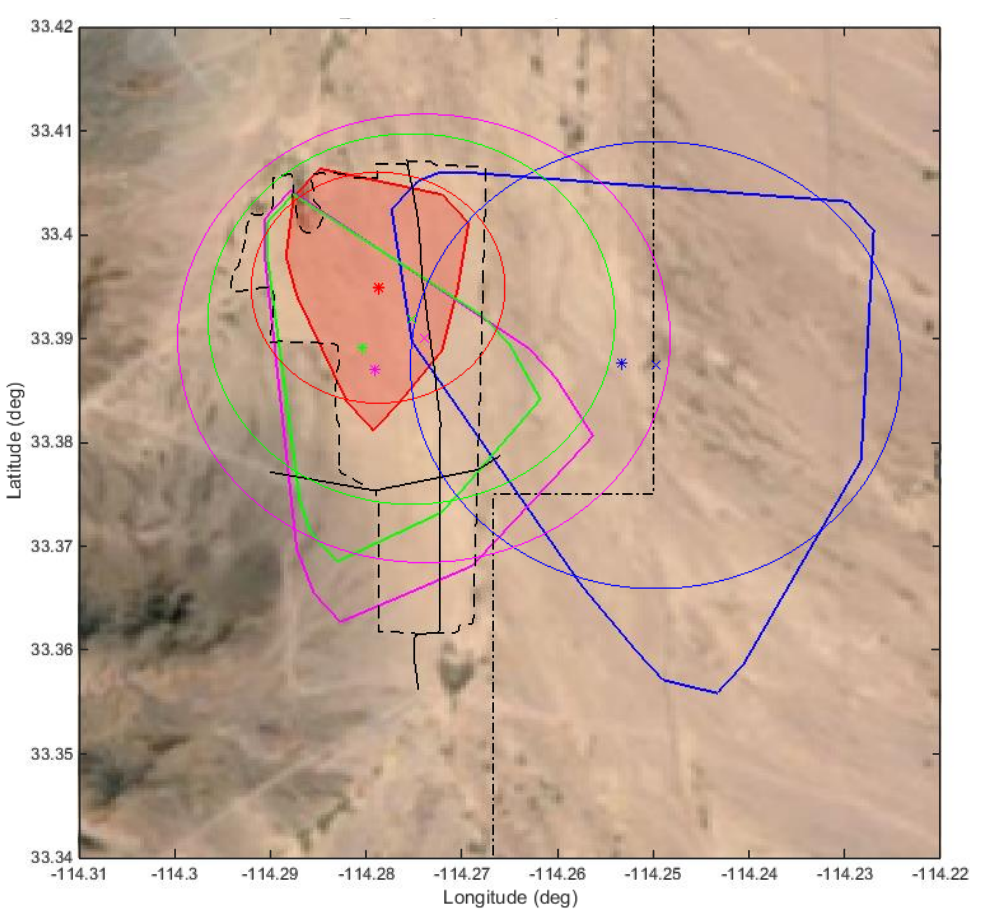

Figure 4. CDT-3-4 footprint comparison: Circles versus Polygons 
Similar comparisons were conducted for other tests. These comparisons provided confidence that Sasquatch Polygons would meet the needs of CPAS to ensure the safety of the test and all assets.

\section{Post-Test Footprint Comparison with Actual Landing Locations}

Pre-flight predictions are only as good as the inputs used. Wind data used for the predictions may be two or three hours old by the time the test is performed. Additionally, the test vehicle may not be released from the aircraft exactly at the prescribed release point. To determine the accuracy of Sasquatch Polygons predictions, footprints were generated for a set of drop tests using weather data acquired just after the time of the tests and the actual release points. The resulting footprints were compared to the actual landing locations. If the actual landing location fell within the corresponding footprint, the prediction was deemed successful.

For every CPAS EDU drop test studied, all of the objects landed within their respective footprints. Most of the parachutes landed close to the nominal, or undispersed, predictions, while the mortar lids and sabots sometimes landed closer to the footprint boundaries. This study showed that Sasquatch Polygons is sufficiently accurate to provide a good representation of the actual trajectory of footprint objects.

\section{Performance During Test Operations}

Sasquatch Polygons has been used successfully as the primary footprint tool on eleven tests to date. The majority of objects land within their dispersion fans, often close to the nominal predicted locations. Objects that present a less consistent aerodynamic shape, such as mortar components, are harder to predict and sometimes fall outside their preflight predicted footprint bounds. Since these objects are too small to visually track, there is no data to use to better understand their trajectories and descent rates. Predicted landing locations are provided to the recovery teams to aid in locating the released objects.

\section{Interface with the Debris Tool}

CPAS drop tests include the requirement for extensive photography and videography of all phases of the test. To best acquire this imagery, several aircraft are staged at different altitudes to observe the test from desired directions. As many as four chase aircraft have been in the air simultaneously during CPAS drop tests. One of the primary chase aircraft is a UH-47 helicopter. Since it is positioned at the main parachute deploy altitude, it is especially susceptible to being struck by objects released from the test vehicle during flight. There is a need to obtain the best quality imagery by getting as close to the payload as possible without compromising safety.

To accomplish this objective, software programmers from a separate NASA project have developed the Debris Tool (DT) as a complement to Sasquatch. The DT graphically displays footprints in real-time relative to the helicopter position. The primary input to ACES DT is a set of trajectory files for each debris object from Sasquatch Polygons. On the morning of a test, once the payload release point has been established, a set of trajectory files is provided to the helicopter crew and used as an input to DT running on an on-board laptop. A GPS unit is connected to the laptop, providing the helicopter's real-time location to DT for plotting on the map alongside the predicted footprint debris field. The helicopter spatial location is used to determine which debris objects may be a danger at any given time. DT allows the helicopter pilot to make any necessary flight-path adjustments if the aircraft was to get too close to any of the high risk regions.

\section{Additional Improvements}

\section{A. Setup File}

Sasquatch Polygons uses a wrapper function into which the user can input all of the information necessary to run the tool. This information includes the parametric sweep to be used, the name and location of the weather file, aircraft release point specification, and options for different modes (e.g. generation of DT input files or running without a windpack). This wrapper allows the tool to be run with minimal user interaction. It also improves clarity of the settings that were used for the most recent run.

\section{B. Enhanced Communication}

The CPAS test team uses results from the footprint tool to make decisions regarding where to extract the test article from the airplane, where to stage the nearby observers, and whether to obtain a waiver for objects crossing the neighboring public highway. Sasquatch Polygons has an option to automatically generate a report with the information needed to make these decisions. This capability minimizes the number of tasks that the user must perform. A commonly overlooked complexity in real-time operations is the stress that the operator can be under. A high priority

American Institute of Aeronautics and Astronautics 
during Sasquatch Polygon development was to reduce the workload. From this effort, a report that summarizes all test-critical Sasquatch information is generated automatically. The report is printed and used to communicate the information to all stakeholders for each hourly weather balloon. Additional new information in the reports includes minimum and maximum predicted times of flight, as well as pressure altitudes for chase aircraft.

Sasquatch maps are now overlaid on a satellite image of the drop zone. This provides more visual information to the test team and YPG personnel.

\section{Changeable Aircraft Heading}

All CPAS drop tests performed on the LaPosa DZ at YPG have been done with the aircraft flying south to north. However, the option may be available to use different headings if an undesirable footprint can be improved by doing so. The heading was recently made an input into the tool; it is set to $0^{\circ}$ (northerly) by default.

\section{Wind Profile Check}

Before the trajectories are propagated, the wind profile is plotted for verification. The user can coplot another profile (e.g. from an earlier weather balloon or forecast) to see the differences and ensure that the current file is not suspect. If the data looks suspect, the user has the option to terminate the run.

\section{Conclusion}

The increased complexity of CPAS testing requires a higher fidelity footprint tool to ensure range safety while allowing for the greatest flexibility in testing. Sasquatch Polygons was developed from the original Sasquatch footprint tool to provide improved trajectory propagation and more realistic dispersions. The interface between Sasquatch Polygons and the chase helicopter represents a significant step forward in range safety. Sasquatch Polygons is currently the primary footprint tool for CPAS testing.

American Institute of Aeronautics and Astronautics 